\title{
Targeting the Monocytic-Endothelial-Platelet Axis with Maraviroc and Pravastatin as a Therapeutic Option to Treat Long COVID/ Post-Acute Sequelae of COVID (PASC)
}

\author{
Bruce Patterson ( $\square$ brucep@incelldx.com ) \\ Ram Yogendra \\ Jose \\ Guevara-Coto \\ Rodrigo \\ Mora-Rodriguez \\ Eric Osgood \\ John Bream \\ Purvi Parikh \\ Mark Kreimer \\ Gary Kaplan \\ Michael Zgoda
}

\section{Research Article}

Keywords: PASC, long COVID, maraviroc, pravastatin, fractalkine, CCR5

Posted Date: February 10th, 2022

DOI: https://doi.org/10.21203/rs.3.rs-1344323/v1

License: @ (i) This work is licensed under a Creative Commons Attribution 4.0 International License. Read Full License 


\section{Abstract}

Post-acute sequelae of COVID (PASC), or long COVID, is a multisystem complication of SARS-CoV-2 infection that continues to debilitate millions worldwide thus highlighting the public health importance of identifying effective therapeutics to alleviate this illness. The pathophysiology behind PASC may be attributed to the recent discovery of persistent S1 protein subunit of SARS-CoV-2 in CD16+ monocytes up to 15 months after infection. CD16+ monocytes, which express both CCR5 and fractalkine receptors (CX3CR1), play a role in vascular homeostasis and endothelial immune surveillance. We believe targeting these receptors using the CCR5 antagonist, maraviroc, along with pravastatin, could disrupt the monocytic-endothelial-platelet axis that may be central to the etiology of PASC. Using five validated clinical scales (NYHA, MRC Dyspnea, COMPASS-31, modified Rankin, and Fatigue Severity Score) to measure 18 participants' response to treatment, we observed significant clinical improvement in six to twelve weeks on a combination of maraviroc 300mg PO BID and pravastatin $10 \mathrm{mg}$ PO daily. Subjective neurological $(p=0.002)$, autonomic $(p<0.0001)$, respiratory $(p=0.0153)$, cardiac $(p=0.002)$ and fatigue $(p<0.0001)$ symptoms scores all decreased which correlated with statistically significant decreases in vascular markers SCD40L and VEGF. These findings suggest that by interrupting the monocytic-endothelial-platelet axis, maraviroc and pravastatin may restore the immune dysregulation observed in PASC and could be potential therapeutic options. This sets the framework for a future double-blinded, placebo-controlled randomized trial to further investigate the drug efficacy of maraviroc and pravastatin in treating PASC.

\section{Introduction}

Post-acute sequelae of COVID (PASC), commonly referred to as long COVID or chronic COVID, is an emerging public health syndrome that continues to devastate and debilitate adult and pediatric survivors of acute SARS-CoV-2 infection. The World Health Organization (WHO)-led Delphi consensus defined PASC as a syndrome starting three months from onset of probable infection with symptoms lasting over two months and could not be explained by an alternative diagnosis (1). Over 200 symptoms have been attributed to PASC (2,) thus posing an enormous challenge clinically. The multi-organ involvement causes cognitive impairment, debilitating neuropathy, chronic migraines, autonomic dysfunction, cardiac dysrhythmias, dyspnea at rest, severe fatigue, and myalgias (3). Presently, minimal therapeutic options are available to treat PASC which can be attributed to the pathology not yet being fully described. However, we recently reported that the S1 protein subunit of SARS-CoV2 is retained in both nonclassical (CD14CD16+) and intermediate (CD14+CD16+) monocytes several months after acute infection. Typically, these monocytes persist only for a few days, but in PASC patients, the S1 containing monocytes can persist for months and years (4), which we propose contributes to the pathophysiology behind PASC. Nonclassical monocytes are involved in phagocytosis and vascular adhesion by patrolling the endothelium under homeostatic and inflammatory conditions through B2 integrin, lymphocyte function-associated antigen-1 (LFA-1) and high levels of fractalkine receptors (CX3CR1) $(5,6)$. On the other hand, CD14+CD16+ monocytes express high levels of C-C chemokine receptor type 5 (CCR5) and fractalkine receptors and are involved in antigen presentation, cytokine secretion and apoptosis regulation $(6,7)$. Since CCR5 and fractalkine receptors have been studied for various chronic inflammatory pathologies, we hypothesized that these receptors may also be therapeutic targets for PASC. CD16+ monocytes also produce high levels of various pro-inflammatory cytokines which could be an explanation for the heterogenous symptomatology in PASC. Specifically, elevations in C-C chemokine ligand 5 (CCL5) /RANTES (Regulated on Normal T-cell Expression and Secretion), IL-2, IL-6, IFN-gamma and Vascular Endothelial Growth Factor (VEGF), along with decrease in CCL4 have been observed in patients and are hypothesized to be contributing to the pathophysiology of PASC (8).

Here, we describe an 18 participant case series investigating the combination of the CCR5 receptor antagonist maraviroc, and pravastatin, which targets fractalkine, as a potential therapeutic approach in addressing and treating 
the potential pathology of PASC. The CCR5 receptor is a seven-transmembrane G protein-coupled receptor (GPCR) that is found on macrophages and T-lymphocytes and functions to regulate trafficking and effector functions of these cells (9). The role of CCR5 as a co-receptor for human immunodeficiency virus (HIV) entry was discovered in 1996. Maraviroc is the first and only US Food and Drug Administration (FDA) and European Medical Agency (EMA) approved CCR5 receptor antagonist available to date. Maraviroc is a negative allosteric modulator of the CCR5 receptor, and by binding to the CCR5 receptor, it induces receptor conformational changes that prevent the chemokine binding of RANTES (CCL5) and CCR5-mediated signaling (10). While this mechanism has been researched and studied extensively in HIV infection, there is increasingly greater recognition and appreciation of the CCR5-CCL5 axis in many other conditions and pathologies such as cancer, autoimmune disorders and endothelial dysfunction. This signaling is central to the pathophysiology of inflammation by directing immune cells through a process called chemotaxis. These actions are mediated through RANTES, which is produced by platelets, macrophages, eosinophils, fibroblasts, endothelial, epithelial and endometrial cells. (11). The effects of RANTES have been implicated in respiratory tract infections, especially viruses possessing RNA genome (including coronavirus, influenza, RSV and adenovirus), asthma, neuroinflammation, and atherosclerosis $(12,13)$. Maraviroc has also been documented to restore the homeostasis of regulatory T-cells (Treg), increase CD4 and CD8 positive counts, and inhibit HIV-associated chronic inflammation and activation $(14,15)$. Interestingly, CD 4 and CD 8 positive T-cells expressing PD-1 and T-regs have been observed to be significantly lower in PASC patients compared to healthy controls (8), thus suggesting maraviroc could restore the immune dysregulation seen in PASC. The commonly known mechanism of action of statins is inhibition of hydroxymethylglutaryl-CoA (HMG-CoA) reductase enzyme in lowering cholesterol. However, statins have also been implicated in reducing inflammation, suppressing fractalkine, and lowering VEGF and IL-6 (16), and as such, may play a role in the pathophysiology of PASC. We targeted fractalkine using pravastatin since CD16+ monocytes express high levels of the fractalkine receptor believing this may address the elevations in vascular markers seen in PASC.

\section{Methods/material}

After written informed consent was obtained, the medical records and immunological lab reports from 17 adult and one pediatric PASC participants from the Chronic COVID Treatment Center treated with maraviroc 300mg PO BID daily and pravastatin 10mg PO daily were collected and analyzed.

\section{Inclusion Criteria}

All the participants in the case series were COVID-19 survivors with documented FDA EUA approved RT-PCR SARSCoV2 positive test and/or were positive for anti-SARS-CoV2 antibodies using FDA EUA approved tests. All participants had one or more new onset symptoms that persisted greater than three months after the diagnosis of acute COVID-19 infection. These symptoms included cognitive impairment (brain fog), migraines, post exertional malaise (PEM), myalgias, arthralgias, severe fatigue, tachyarrhythmias, postural orthostatic tachycardia syndrome (POTS) and shortness of breath. All participants displayed either isolated or combinations of elevated pro-inflammatory markers: RANTES, TNF-alpha, IFN-gamma, sCD40L, VEGF, IL-6, IL-2 and IL-8 on the IncellKINE panel. The IncellKINE cytokine panel is a set of 14 cytokines that was constructed from a machine-based learning algorithm that identified potential markers of PASC.

\section{Exclusion Criteria}

We excluded participants with a history of migraines, neuropathy, inflammatory bowel disease, depression and anxiety disorders, chronic fatigue syndrome, fibromyalgia, arthritis, COPD, asthma, chronic kidney disease, chronic heart failure (CHF), arrhythmias, bleeding disorders, and anticoagulation therapy prior to COVID-19 infection. 


\section{Validated Scoring System for Patient Assessment Before and After Treatment}

A challenge in studying and defining PASC is the heterogenous clinical presentation and multisystem involvement. Thus, we categorized the main participant symptoms into 5 groups: neurological/autonomic function, cardiac, respiratory, overall functionality and fatigue. Since there are no validated scales for PASC, we used five validated scales for other organ systems (New York Heart Association (NYHA), Modified Rankin Scale for Neurologic Disability, Fatigue Severity Scale (FSS), COMPASS-31 and Medical Research Council (MRC) Dyspnea Scale, respectively) to measure subjective participant responses to treatment. Participants were administered validated self-questionnaires about their PASC symptoms before and after treatment with maraviroc and pravastatin treatment. The length of duration of treatment varied based on repeat immune markers and participant-reported symptom improvement. Since many of these participants were on other medications and anti-inflammatories prior to starting maraviroc and pravastatin, the biomarkers and subjective data presented are from the onset of this combination. Phone interviews were conducted with each participant before and after subjective responses to the medications.

The New York Heart Association (NYHA) Functional Classification was used to classify severity of PASC associated cardiac symptoms.

\begin{tabular}{|c|c|c|c|c|c|c|c|}
\hline $\begin{array}{l}\text { Class } \\
1\end{array}$ & $\begin{array}{l}\text { No limitation of } \\
\text { physical activity. } \\
\text { Ordinary physical } \\
\text { activity does not } \\
\text { cause undue fatigue, } \\
\text { palpitation, dyspnea } \\
\text { (shortness of } \\
\text { breath). }\end{array}$ & $\begin{array}{l}\text { Class } \\
2\end{array}$ & $\begin{array}{l}\text { Slight limitation of } \\
\text { physical activity. } \\
\text { Comfortable at rest. } \\
\text { Ordinary physical } \\
\text { activity results in } \\
\text { fatigue, palpitation, } \\
\text { dyspnea (shortness } \\
\text { of breath). }\end{array}$ & $\begin{array}{l}\text { Class } \\
3\end{array}$ & $\begin{array}{l}\text { Marked } \\
\text { limitation of } \\
\text { physical } \\
\text { activity. } \\
\text { Comfortable } \\
\text { at rest. Less } \\
\text { than ordinary } \\
\text { activity } \\
\text { causes } \\
\text { fatigue, } \\
\text { palpitation, or } \\
\text { dyspnea. }\end{array}$ & $\begin{array}{l}\text { Class } \\
4\end{array}$ & $\begin{array}{l}\text { Unable to } \\
\text { carry on } \\
\text { any } \\
\text { physical } \\
\text { activity } \\
\text { without } \\
\text { discomfort. } \\
\text { Symptoms } \\
\text { of heart } \\
\text { failure at } \\
\text { rest. If any } \\
\text { physical } \\
\text { activity is } \\
\text { undertaken, } \\
\text { discomfort } \\
\text { increases. }\end{array}$ \\
\hline
\end{tabular}

The Composite Autonomic Symptom Scale 31 (COMPASS 31), a self-rating questionnaire consisting of 31 items and evaluating orthostatic intolerance, vasomotor, secretomotor, gastrointestinal, bladder, and pupillomotor function, was used to measure autonomic dysfunction and the subsequent therapeutic effects of maraviroc and pravastatin. A sub raw score for each of the six domains was calculated and converted into a weighted sub-score. The sum of this weighted sub-score gave a total score which ranged from 0 to 100, with 0 meaning no autonomic symptoms and 100 reflecting the most severe autonomic symptoms.

Medical Research Council (MRC) Dyspnea Scale is a validated method comprised of five statements that aims to measure perceived feeling of breathlessness. 


\begin{tabular}{|c|c|c|c|c|c|c|c|c|c|}
\hline $\begin{array}{l}\text { Grade } \\
1\end{array}$ & $\begin{array}{l}\text { Are you ever } \\
\text { troubled by } \\
\text { breathlessness } \\
\text { except on } \\
\text { strenuous } \\
\text { exertion? }\end{array}$ & $\begin{array}{l}\text { Grade } \\
2\end{array}$ & $\begin{array}{l}\text { Are you } \\
\text { short of } \\
\text { breath } \\
\text { when } \\
\text { hurrying } \\
\text { on the } \\
\text { level or } \\
\text { walking } \\
\text { up a } \\
\text { slight } \\
\text { hill? }\end{array}$ & $\begin{array}{l}\text { Grade } \\
3\end{array}$ & $\begin{array}{l}\text { Do you } \\
\text { have to } \\
\text { walk } \\
\text { slower } \\
\text { than } \\
\text { most } \\
\text { people } \\
\text { on the } \\
\text { level? Do } \\
\text { you have } \\
\text { to stop } \\
\text { after a } \\
\text { mile or } \\
\text { so (or } \\
\text { after } 15 \\
\text { minutes) } \\
\text { on the } \\
\text { level at } \\
\text { your own } \\
\text { pace? }\end{array}$ & $\begin{array}{l}\text { Grade } \\
4\end{array}$ & $\begin{array}{l}\text { Do you } \\
\text { have to } \\
\text { stop for } \\
\text { breath } \\
\text { after } \\
\text { walking } \\
\text { about } \\
100 \text { yds. } \\
\text { (or after } \\
\text { a few } \\
\text { minutes) } \\
\text { on the } \\
\text { level? }\end{array}$ & $\begin{array}{l}\text { Grade } \\
5\end{array}$ & $\begin{array}{l}\text { Are you too } \\
\text { breathless } \\
\text { to leave the } \\
\text { house, or } \\
\text { breathless } \\
\text { after } \\
\text { undressing? }\end{array}$ \\
\hline
\end{tabular}

The Modified Rankin Scale for Neurologic Disability is a validated scale to measure degree of disability after suffering a stroke or neurological insult.

\begin{tabular}{|c|c|c|c|c|c|c|c|c|c|c|c|}
\hline 0 & $\begin{array}{l}\text { no } \\
\text { symptoms }\end{array}$ & 1 & $\begin{array}{l}\text { No } \\
\text { significant } \\
\text { disability } \\
\text { despite } \\
\text { symptoms; } \\
\text { able to } \\
\text { carry out all } \\
\text { usual } \\
\text { duties and } \\
\text { activities }\end{array}$ & 2 & $\begin{array}{l}\text { Slight } \\
\text { disability; } \\
\text { unable to } \\
\text { carry out all } \\
\text { previous } \\
\text { activities, } \\
\text { but able to } \\
\text { look after } \\
\text { own affairs } \\
\text { without } \\
\text { assistance }\end{array}$ & 3 & $\begin{array}{l}\text { Moderate } \\
\text { disability; } \\
\text { requiring } \\
\text { some help, } \\
\text { but able to } \\
\text { walk } \\
\text { without } \\
\text { assistance }\end{array}$ & 4 & $\begin{array}{l}\text { Moderately } \\
\text { severe } \\
\text { disability; } \\
\text { unable to } \\
\text { walk } \\
\text { without } \\
\text { assistance } \\
\text { and unable } \\
\text { to attend to } \\
\text { own bodily } \\
\text { needs } \\
\text { without } \\
\text { assistance }\end{array}$ & 5 & $\begin{array}{l}\text { Severe } \\
\text { disability; } \\
\text { bedridden, } \\
\text { incontinent } \\
\text { and } \\
\text { requiring } \\
\text { constant } \\
\text { nursing } \\
\text { care and } \\
\text { attention }\end{array}$ \\
\hline
\end{tabular}

The Fatigue Severity Scale (FSS) questionnaire is a nine-statement validated scale that rates the severity of fatigue symptoms. Participants were asked how accurately each statement reflected their condition before and after treatment with maraviroc and pravastatin and the extent to which they agreed or disagreed based on a scale of 1 (strongly disagree) to 7 (strongly agree).

\begin{tabular}{|c|c|c|c|c|c|c|c|c|c|c|c|}
\hline 1 & $\begin{array}{l}\text { My motivation } \\
\text { is lower when I } \\
\text { am fatigued. }\end{array}$ & 2 & $\begin{array}{l}\text { Exercise } \\
\text { brings on } \\
\text { excessive } \\
\text { fatigue. }\end{array}$ & 3 & $\begin{array}{l}\text { I am } \\
\text { easily } \\
\text { fatigued. }\end{array}$ & 4 & $\begin{array}{l}\text { Fatigue } \\
\text { interferes } \\
\text { with my } \\
\text { physical } \\
\text { functioning. }\end{array}$ & 5 & $\begin{array}{l}\text { Fatigue } \\
\text { causes } \\
\text { frequent } \\
\text { problems } \\
\text { for me. }\end{array}$ & 6 & $\begin{array}{l}\text { My fatigue } \\
\text { prevents } \\
\text { sustained } \\
\text { physical } \\
\text { functioning }\end{array}$ \\
\hline 7 & $\begin{array}{l}\text { Fatigue } \\
\text { interferes with } \\
\text { carrying out } \\
\text { certain duties } \\
\text { and } \\
\text { responsibilities. }\end{array}$ & 8 & $\begin{array}{l}\text { Fatigue is } \\
\text { among my } \\
\text { three most } \\
\text { disabling } \\
\text { symptoms. }\end{array}$ & 9 & $\begin{array}{l}\text { Fatigue } \\
\text { interferes } \\
\text { with my } \\
\text { work, } \\
\text { family, or } \\
\text { social } \\
\text { life. }\end{array}$ & & & & & & \\
\hline
\end{tabular}


Fresh plasma was used for cytokine quantification using a customized 14-plex bead based flow cytometric assay (IncellKINE, IncellDx, Inc) on a CytoFlex flow cytometer as previously described (8) using the following analytes: 'TNFa', 'IL-4', 'IL-13','IL-2', 'GM-CSF', 'sCD40L', 'CCL5 (RANTES)', 'CCL3 (MIP-1a)','IL-6', 'IL-10', 'IFN- $y$ ', 'VEGF', 'IL-8', and 'CCL4 (MIP-1 $\beta$ ). For each participant sample, $25 \mu \mathrm{L}$ of plasma was used in each well of a $96-$ well plate.

\section{Data acquisition and preprocessing}

The dataset was acquired in a Microsoft Excel (xlsx) table format, consisting of a total of 22 columns representing different features. The features or columns were organized in the following order:

- Anonymized participant ID (column 1)

- Weeks on medication (column 2)

- Status of participant - before or after treatment (column 3)

- Cytokine biomarker profiles (columns 4-17)

- Subjective scores (columns 18-22)

In total there were 18 unique individuals, with each individual being represented in duplicate for before and after treatment. The presence of a pre and post treatment for each individual categorized as PASC allowed us the possibility to separate the data set into a before and after data sets for the required statistical comparisons. To separate the before and after groups, we used the python programming language (version 3.9) and the pandas library $(18,19)$, which allowed us to group the samples according to before and after treatment. Once we separated the data in the two data sets, we then conducted the necessary comparative statistical analysis, including the statistical test to determine if there were significant differences between the two groups.

\section{Wilcoxon's paired test to Compare the before and after treatment groups}

To determine if there were differences between the biomarker's levels of the two groups (before and after) we decided to compare the datasets by implementing the non-parametric Wilcoxon's paired test. The implementation of this test was done using the python library scipy (20). The selection of the Wilcoxon test was based on the assumption that this non-parametric test does not assume normal distribution of the variables. Additionally, in contrast to parametric tests like ANOVA, Wilcoxon's paired test does not base its comparison on the mean but median values. For our data we compared group before and group after with two alternative hypotheses. The first was a two-sided test, which resulted in a p-value less than 0.05 . Subsequently, we tested for an alternative hypothesis "greater", resulting in a pvalue of less than 0.05 .

\section{Correlation analysis between biomarker levels and subjective scores}

In order to identify potential statistically significant relationships between the biomarkers present in the dataset and the subjective scores, we imported the full dataset into the R programming language (version 4.1.1) (21) and conducted a correlation analysis. The correlation analysis was calculated using the Pearson correlation coefficient, which allows the measurement of both strength and direction of the linear relationship between two variables.

The Pearson correlation coefficient has the advantage that its values are highly interpretable, always ranging from - 1 (strong negative correlation) to +1 (strong positive correlation). Correlation coefficients were calculated for both the before and after data points, and to validate their statistical significance, their p-value was calculated. We defined that correlation coefficients were statistically significant if their p-value was equal or less than 0.05 . In order to properly 
interpret and convey the correlation relationships and their statistical significances, we constructed a modified pair plot with the R package GGally and additional functions to plot the p-values for the correlation coefficients. GGally is an extension to the $\mathrm{R}$ package ggplot2 (versions 2.1.2 and 3.3.5 respectively) (22). The pair plot presented was color coded for each group (blue = before, red = after) and consisted of scatterplots of each variable in the dataset for both the before and aftergroups, a density plot (a smooth representation of a histogram to approximate the distribution of each group), and the correlations for each group as well as the joint correlation. For the correlation coefficients, the pvalues were added under the Pearson's correlation coefficient and maintain the color code scheme, with the addition of black representing the joint correlation.

\section{Validation of long hauler status using a machine learning classifier}

The individuals in the dataset were identified as a long hauler (someone diagnosed with PASC). In order to further validate this classification, we implemented our previously reported machine learning classifier (8) using both the before and after datasets as prediction sets for the model to label. In brief, this random forest was constructed using a dataset composed of 4 classes (control individuals, mild-moderate cases, severe and PASC individuals). Because of the unbalanced nature of the dataset, the training set was subjected to a minority class balancing method that generates synthetic samples by means of interpolation (SMOTE) (23). Prediction of the labels was done once the model was fine-tuned, using an unseen test set, which was not subjected to SMOTE to avoid contamination or overfitting. We used this model to predict the labels of both groups in order to further validate the classification/labeling of the dataset individuals as PASC.

\section{Results}

\section{Comparison between "before" and "after" treatment demonstrates statistical differences between groups}

The statistical comparison using the Wilcoxon paired test to contrast the before and after treatment groups using a two-sided alternative hypothesis revealed the existence of statistically significant differences between the cytokine profiles (biomarkers) between the before and after treatment groups ( $p$-value $=2.20 \mathrm{e}-17$ ). For this test, group 1 was before and group 2 was after. The results of the Wilcoxon test support that the medians of both groups are different and that a one-tailed test needed to be done. Based on the results of the two-sided test, we proceeded to do a onesided. The alternative hypothesis of this second test was focused on determining if the medians values for the biomarkers in treatment group 1 (before) were greater than those of group 2 (after). This test resulted in a statistically significant difference, where the $p$-value was less than the threshold of 0.05 ( $p$-value $=1.10 \mathrm{e}-17$ ).

Our results indicate that the biomarker (cytokine profiles) of the individuals from individuals in the dataset before treatment are, statistically different from those after treatment. Moreover, our statistical test suggests that for these individuals, these biomarkers are statistically greater before treatment.

\section{Correlation analysis indicates the presence of positive correlations between cytokine biomarkers and subjective scores}

We constructed a correlation matrix using the Pearson's correlation coefficient in order to identify the positive correlations between the different biomarkers (cytokines) in the dataset and the subjective scores present. We calculated three correlation coefficients. The first is the joint correlation, which represents the relationship between the full dataset (both before and after treatment groups), followed by the coefficients for each treatment group, as shown 
in Figure 1 (correlation matrix). In addition to the correlation coefficient, we calculated the corresponding p-value to support the statistical significance of these relationships. We defined our significance threshold to $p$-values of $\leq 0.05$.

We analyzed the linear relationship between the cytokine biomarkers and the modified Rankin score (24). In brief, this is a 6-point disability scale that ranges from 0 (individual has no residual symptoms) to 5 (the individual is bedridden, incontinent and requires continuous care). According to the documentation an additional value of 6 is included for deceased or "expired" individuals. For the Rankin subjective score, we identified a low positive correlation with statistical significance for two biomarkers, VEGF and SCD40L (Figure 2). Finally, we did the correlation analysis for the COMPASS 31 score (25). This scale was developed as a robust statistical instrument to determine autonomic symptoms, thus providing relevant severity scores for clinical assessment. For this scale, we identified that several cytokines had statistically significant relationships to the subjective score. TNF-alpha and GM CSF had low positive correlations, while VEGF and SCD40L showed moderate positive correlation (Figure 3).

\section{Figure 4: New York Heart Association Classification}

For it is possible to note that for the first subjective score, the New York Heart Association (NYHA) Functional Classification, which labels individuals in one of four categories, we were able to identify two statistically significant biomarkers in the joint correlation (Figure 4). The cytokines IL-8 and GM-CSF showed a low positive correlation to the NYHA score, with both having $r$ values between 0.30 and 0.50 . The linear association between IL-8 and GM-CSF indicates that there appears to be a weak linear association between both treatment groups (before and after) where the levels of both cytokines appear to be positively associated with the NYHA score.

\section{Figure 5: Medical Research Council (MRC) Dyspnea Scale}

When subsequently analyzed the correlation values for the Medical Research Council (MRC) Dyspnea scale score (Figure 5), which is a simple scale allowing participants to indicate the effects of breathlessness on mobility, we were able to identify that for both treatment groups (joint correlation), the biomarkers GM-CSF, TNF-alpha and SCD40L presented statistically correlations. In the case of GM-CSF, the linear association between the cytokine and the subjective score was $\mathbf{0 . 5 9 3}$, which makes it a moderate positive correlation. For TNF-alpha and SCD40L there correlation values were in ranges between 0.30 and 0.50 , indicating their association with the MRC Dyspnea score were low positive.

\section{Figure 6: Fatigue Severity Score (FSS)}

In addition, the correlation analysis of the Fatigue score from the Shirley Ryan Ability Lab at the Rehabilitation Institute of Chicago (https://www.sralab.org/rehabilitation-measures/fatigue-severity-scale) provides a 9-item scale allowing the measurement of the effects of fatigue on an individual. The scores range from a value of 9 (lowest possible score) to 63 (highest fatigue effects). Our analysis identified that various biomarkers showed statistically significant correlations (Figure 6). These linear associations were present in both the before and aftertreatment groups (joint correlation). The cytokines IL-2, SCD40L, TNF alpha and VEGF presented a positive correlation, with $r$ values ranging between 0.50 and 0.70 , as shown in Figure 1. In addition to these biomarkers, IL-8, IL-10 and GM CSF presented low positive correlations, with $r$ values ranging between 0.30 and 0.50 .

Our results suggest that there are a number of biomarkers that appear to be positively associated in varying degrees with the various subjective scores. The most common cytokine was SCD40L, positively associated to all scores except for the NYHA Functional Classification score. Another interesting finding is the relationship of GM-CSF to a wide variety of subjective scores. This cytokine had significant positive association to all scales except for the modified 
Rankin score. Finally, both VEGF and TNF-alpha were correlated with 3 of the 5 subjective scores, with VEGF not having a significant relation to NHYA and MRC Dyspnea, while TNF-alpha not correlating to NYHA and Rankin. These results suggest that many cytokine biomarkers possess for both the before and after treatment groups positive levels of statistically significant relationship.

Machine learning classifier validates the labelling of individuals in the dataset group as PASC using cytokine profiles

The individuals in the dataset were identified as being composed of long hauler or PASC individuals. In order to validate this assessment, we used the previously published random forest classifier (6) to label each of the treatment groups. The 36 instances (18 individuals for each treatment) were identified as belonging to the PASC class, according to the model. This classification was of great importance because it allowed us to use the long hauler/PASC heuristic score previously developed in (6) to further understand how these individuals have altered their behavior before and after treatment.

\section{Discussion}

The discovery of CD16+ monocytes containing persistent S1 proteins from PASC patients may help further understand its pathophysiology and identify targets for therapy (4). Both CD16+ monocytes subsets, intermediate (CD14+CD16+) and nonclassical (CD14- CD16+), respectively, are known to interact significantly with the endothelium and platelets via the fractalkine pathway (26). This suggests that the pathophysiology of PASC may lie with the monocytic-endothelial-platelet axis. Fractalkine, which mediates cell adhesion and leucocyte recruitment, is a transmembrane protein expressed in the brain, colon, heart, and lung, along with endothelial cells and astrocytes. Intermediate monocytes express high levels of both CCR5 and fractalkine receptors, whereas nonclassical monocytes express high levels of fractalkine receptors $(6,7)$. This interaction between fractalkine and fractalkine receptors have been involved in the pathogenesis of atherosclerosis, vasculitis, vasculopathies, and inflammatory brain disorders (27) and could also be contributing to vascular endothelialitis in PASC. Vascular endothelialitis leads to collagen exposure along with platelet activation and adherence via glycoprotein 1b-IX-V-receptor (GPIb-IX-V) with collagenbound von Willebrand factor (VWF) (28). Activated platelets release soluble CD40 ligand (sCD40L) to recruit both neutrophils and monocytes to the vascular lesions (29), thus activating the coagulation cascade. Stimulated platelets also release RANTES which binds to endothelial cells and encourages monocyte adhesion to inflamed endothelial tissues (30) and acts as a chemotactic agent for inflammatory cells. Activated platelets and endothelial cells can also secrete VEGF which induces angiogenesis and microvascular hyperpermeability. VEGF is a diagnostic marker for vasculitic neuropathy and also contributes to a pro-inflammatory-prothrombotic environment (31). While the vascular effects of statins have been well documented (32), the protective role of maraviroc on the endothelium has also been similarly published (33). Hence, we targeted CCR5 and fractalkine receptors on the S1 protein expressing CD16+ monocytes using maraviroc and pravastatin, respectively, hypothesizing that this combination could be therapeutically effective in treating vascular endothelialitis and resolving symptoms associated with PASC.

Neurological symptoms associated with PASC include severe headaches and cognitive impairment (brain fog), along with neuropathy and weakness, necessitating the need for assistance in performing daily tasks. CD14+CD16+ monocytes are known to transmigrate across the blood brain barrier and play an important role in central nervous system (CNS) immune surveillance. These monocytes were implicated as HIV reservoirs in the CNS causing neuroinflammation, neuronal damage, and cognitive defects (34). We hypothesize that the S1 protein containing CD14+CD16+ monocytes in PASC patients are also crossing the blood brain barrier and triggering neuroinflammation and inducing neurological symptoms. Both maraviroc and statins are known to cross the blood-brain-barrier, and more specifically, maraviroc has been suggested as treatment for Parkinson's, neurocognitive impairment, and strokes (35). 
Interestingly, after the introduction of maraviroc and pravastatin, participants showed a decrease in modified Rankin scale scores $(p=0.0002)$ (Figure 2 ) and reported improvement in neurological function and ability. These findings were correlated with a statistically significant decrease in VEGF $(r=0.4, p=0.02)$ and SCD40L $(r=0.42, p=0.01)$, suggesting treatment targeting cytokines associated with vascular endothelialitis correlated with improvement in neurological symptoms.

Autonomic dysfunction such as postural orthostatic tachycardia syndrome (POTS) and light sensitivity has also been associated with PASC. POTS is a syndrome consisting of unexplained tachycardia, dizziness, light-headedness, fainting, and abdominal pain. While the true etiology of POTS has yet to be defined, endothelial dysfunction has been suggested as the pathophysiology (36). There is also evidence that POTS maybe be associated with G-proteincoupled receptor autoantibodies (37). Interestingly, since CCR5 and fractalkine receptor are also G-protein-coupled receptors $(9,38)$, it is possible that antagonism of these receptors could also inhibit the autonomic effects of these autoantibodies. We observed a statistically significant decrease in COMPASS-31 ( $p=0.0001$ ) (Figure 3 ) scores correlating with statistically significant decreases in VEGF ( $r=0.6, p=0.0005)$, sCD40L $(r=0.6, p=0.0001)$, and TNFalpha $(r=0.5, p=0.0026)$, suggesting that pro-inflammatory macrophage activation may be triggering vascular endothelialitis. Interestingly, elevations in SCD40L have also been associated with sympathoadrenal activation and targeting these vascular markers may address PASC associated dysautonomia (39).

Cardiorespiratory complaints such as chest pain, shortness of breath, and symptoms resembling POTS are very commonly reported by PASC patients. Many PASC patients with cardiac and pulmonary symptoms have undergone extensive workup (EKG, echocardiogram, stress test, pulmonary function testing, etc.) which have not detected any abnormalities or pathologies. Subsequently, current clinical approaches have only been used to treat symptoms with antiarrhythmics, bronchodilators or alpha-adrenergics, instead of addressing the underlying pathophysiology. We observed an improvement in cardiac symptoms evidenced by a decrease in NYHA functional classification $(p=0.002)$ (Figure 4). This improvement was associated with statistically significant decreases in IL-8 ( $r=0.4, p=0.03)$ and GM$\operatorname{CSF}(r=0.4, p=0.01)$. Interestingly, endothelial cells are main producers of IL-8 (40) and statins are known to decrease IL-8 (41). Additionally, maraviroc has been suggested as reducing the cardiovascular risk for acute coronary disease by protecting the endothelium from pro-inflammatory macrophage infiltration (42). These mechanisms potentially support their use in addressing PASC associated cardiac symptoms. We also observed improvement in respiratory symptoms after initiating maraviroc and pravastatin therapy. Participants reported improvements as reflected by a statistically significant decrease in the MRC Dyspnea scale $(p=0.0153)$ (Figure 5$)$. These responses and improvements correlated with statistically significant decreases in IL-2 ( $r=0.4, p=0.05), G M-C S F(r=0.6, p=0.0002), s C D 40 L(r=0.4$, $p=0.04)$, and TNF-alpha $(r=0.4, p=0.01)$. Intriguingly, CD16+ monocytes are known to produce large quantities of TNFalpha and could be activated by the retained S1 proteins (43), causing vascular endothelialitis via the fractalkinefractalkine receptor interaction in pulmonary vasculature. Elevations in SCD40L have been associated with pulmonary arterial hypertension (PAH) (44), while IL-2 can induce pulmonary microvasculature injury and generate an asthma-like bronchoconstriction (45). We previously published a multi-class model score that described an increase IL-2 as a characteristic specific to PASC (8), thus confirming the clinical significance of IL-2 in PASC. Both maraviroc and statins can decrease IL-2 and TNF-alpha $(41,46)$, which may explain the observed improvements in PASC associated respiratory symptoms. The patient Fatigue Severity Score (FSS) also significantly decreased $(p<0.0001)$ (Figure 6) after maraviroc and pravastatin which correlated with decrease in SCD40L $(r=0.5, p=0.001)$, VEGF $(r=0.5, p=0.001)$, TNF-alpha $(r=0.7, p=4 e-5)$, IL-2 ( $r=0.6, p=0.0005)$, and GM-CSF $(r=0.5, p=0.004)$, again suggesting that targeting the monocytic-platelet-endothelial axis can alleviate PASC associated fatigue.

Despite a black box warning for hepatoxicity, maraviroc has demonstrated a strong safety profile in adult, pediatric, and neonatal populations $(47,48)$. Analysis of the MOTIVATE study demonstrated a low incidence of hepatoxicity 
with maraviroc even after 96 weeks of treatment at the FDA approved dose of 300mg B.I.D (49). This influenced our decision to treat with this dose. Hepatic safety was monitored in all the participants by measuring and evaluating AST, ALT, and total bilirubin (LFTs) prior to commencing treatment with maraviroc and every two weeks while on treatment. None of participants presented here experienced any clinical signs of hepatotoxicity or elevated liver function serologies while on, or after, treatment. Maraviroc is metabolized by CYP3A4, and we chose to avoid any CYP3A4 metabolizing statins to mitigate any potential drug interactions. This approach guided our decision to treat with pravastatin $10 \mathrm{mg}$ PO daily over the other statins since it is metabolized via glucuronidation. However, the therapeutic benefits with other statins have also been observed and could be considered.

Since some of the participants were already on other therapeutics including ivermectin, fluvoxamine, and prednisone, all the biomarker data and subjective responses were documented from the initiation of maraviroc and pravastatin. Some participants saw symptom relief after six weeks and were ready to stop all medications, while others needed treatment up to twelve weeks before discontinuing medications. Further studies will need to be conducted to understand this variation in length of treatment between participants. The results we present in this case series do not replace the need for a double-blinded placebo controlled randomized trial to understand drug efficacy. However, we do believe this case series sets the framework for such future clinical trial designs to further investigate the efficacy and usefulness of maraviroc and pravastatin to treat PASC.

\section{Declarations}

Acknowledgements: The authors would like to acknowledge the work of Brittany McKenney RN, Amy White RN, Surlin Chadha RN, Amanda Robinson and Christine Meda in assisting in the study and interacting with the patients.

Ethics Statement: All the patients/participants provided their written informed consent to participate in this study.

Funding: None

Author contributions:

RY, EO and MZ conceptualized the study. RY organized the study. JG-C,and RM-R performed the bioinformatics. RY, JG-C,and RM-R wrote the draft of the manuscript. All authors contributed to revising the manuscript and approved the submitted version.

Competing Interests:

BP is an employee of IncellDX.

$\mathrm{BP}, \mathrm{RY}, \mathrm{PP}, \mathrm{JB}, \mathrm{EO}$, and MK are independent contractors of the Chronic COVID Treatment Center.

The remaining authors declare that the research was conducted in the absence of any commercial or financial relationships that could be construed as a potential conflict of interest.

Data and materials availability:

All requests for materials and data should be addressed to the corresponding author.

Ethics: This study was reviewed and approved by the ethics committee of the Chronic COVID Treatment Center.

\section{References}

Page $11 / 18$ 
1. Soriano JB, Murthy S, Marshall JC, Relan P, Diaz JV, WHO Clinical Case Definition Working Group on Post-COVID19 Condition. A clinical case definition of Post-COVID-19 condition by a Delphi consensus. Lancet Infect Dis. Published online December 21, 2021:S1473-3099(21)00703-9.

2. Davis HE, Assaf GS, McCorkell L, et al. Characterizing Long COVID in an International Cohort: 7 months of symptoms and their impact. eClinicalMedicine. 2021;38.

3. Novak P, Mukerji SS, Alabsi HS, et al. Multisystem Involvement in Post-Acute Sequelae of Coronavirus Disease 19. Ann Neurol. Published online December 24, 2021.

4. Patterson BK, Francisco EB, Yogendra R, et al. Persistence of SARS-CoV2-2 S1 Protein in CD16+ Monocytes in Post-Acute Sequelae of Covid-19 (PASC) up to 15 months post-infection. Front Immunol. 2022;0.

5. Thomas G, Tacke R, Hedrick CC, Hanna RN. Nonclassical Patrolling Monocyte Function in the Vasculature. Arteriosclerosis, Thrombosis, and Vascular Biology. 2015;35(6):1306-1316.

6. Kapellos TS, Bonaguro L, Gemünd I, et al. Human Monocyte Subsets and Phenotypes in Major Chronic Inflammatory Diseases. Front Immunol. 2019;0.

7. Weber C, Belge KU, von Hundelshausen P, et al. Differential Chemokine Receptor Expression and Function in Human Monocyte Subpopulations. J Leukoc Biol. 2000;67(5):699-704.

8. Patterson BK, Guevara-Coto J, Yogendra R, et al. Immune-based Prediction of COVID-19 Severity and Chronicity Decoded Using Machine Learning. Front Immunol. 2021;0.

9. Oppermann M. Chemokine receptor CCR5: insights into Structure, Function, and Regulation. Cell Signal. 2004;16(11):1201-1210.

10. Vangelista L, Vento S. The Expanding Therapeutic Perspective of CCR5 Blockade. Front Immunol. 2018;0.

11. Marques RE, Guabiraba R, Russo RC, Teixeira MM. Targeting CCL5 in Inflammation. Expert Opin Ther Targets. 2013;17(12):1439-1460.

12. Patterson BK, Seethamraju H, Dhody K, et al. CCR5 Inhibition in Critical COVID-19 Patients Decreases Inflammatory Cytokines, Increases CD8 T-cells, and Decreases SARS-CoV2 RNA in Plasma by Day 14. International Journal of Infectious Diseases. 2021;103:25-32.

13. Bowen GP, Borgland SL, Lam M, Libermann TA, Wong NCW, Muruve DA. Adenovirus Vector-induced Inflammation: Capsid-Dependent Induction of the C-C chemokine RANTES requires NF-kappa B. Hum Gene Ther. 2002;13(3):367-379.

14. Arberas H, Guardo AC, Bargalló ME, et al. In Vitro Effects of the CCR5 Inhibitor Maraviroc on Human T cell Function. J Antimicrob Chemother. 2013;68(3):577-586.

15. Pozo-Balado MM, Rosado-Sánchez I, Méndez-Lagares G, et al. Maraviroc Contributes to the Restoration of the Homeostasis of Regulatory T-cell Subsets in Antiretroviral-Naive HIV-Infected Subjects. Clin Microbiol Infect. 2016;22(5):461.e1-5.

16. Cimato TR, Palka BA. Fractalkine (CX3CL1), GM-CSF and VEGF-A Levels are Reduced by Statins in Adult Patients. Clin Transl Med. 2014;3:14.

17. Eisinger F, Patzelt J, Langer HF. The Platelet Response to Tissue Injury. Front Med. 2018;0.

18. Model MS-JMG, 1999 undefined. Python: a programming language for software integration and development. academia.edu Available at: https://www.academia.edu/download/25505223/10.1.1.35.6459.pdf [Accessed February 3, 2022]

19. Mckinney W. pandas: a Foundational Python Library for Data Analysis and Statistics. Available at: http://pandas.sf.net [Accessed April 17, 2021] 
20. Virtanen P, Gommers R, Oliphant TE, Haberland M, Reddy T, Cournapeau D, Burovski E, Peterson P, Weckesser W, Bright J, et al. SciPy 1.0: fundamental algorithms for scientific computing in Python. Nat Methods 2020173 (2020) 17:261-272. doi:10.1038/s41592-019-0686-2

21. Ihaka R, Gentleman R. R: A Language for Data Analysis and Graphics. J Comput Graph Stat (1996) 5:299-314. doi:10.1080/10618600.1996.10474713

22. Emerson JW, Green WA, Schloerke B, Crowley J, Cook D, Hofmann H, Wickham H. The generalized pairs plot. J Comput Graph Stat (2013) 22:79-91.

23. Chawla N V., Bowyer KW, Hall LO, Kegelmeyer WP. SMOTE: Synthetic minority over-sampling technique. J Artif Intell Res (2002) 16:321-357. doi:10.1613/jair.953

24. Modified Rankin Score (mRS). Available at: https://manual.jointcommission.org/releases/TJC2018A/DataElem0569.html [Accessed February 3, 2022]

25. Sletten DM, Suarez GA, Low PA, Mandrekar J, Singer W. COMPASS 31: a refined and abbreviated Composite Autonomic Symptom Score. Mayo Clin Proc (2012) 87:1196-1201. doi:10.1016/J.MAYOCP.2012.10.013

26. Kasama T, Wakabayashi K, Sato M, Takahashi R, Isozaki T. Relevance of the CX3CL1/Fractalkine-CX3CR1 Pathway in Vasculitis and Vasculopathy. Transl Res. 2010;155(1):20-26.

27. Thomas G, Tacke R, Hedrick CC, Hanna RN. Nonclassical Patrolling Monocyte Function in the Vasculature. Arteriosclerosis, Thrombosis, and Vascular Biology. 2015;35(6):1306-1316.

28. Varga-Szabo D, Pleines I, Nieswandt B. Cell Adhesion Mechanisms in Platelets. Arteriosclerosis, Thrombosis, and Vascular Biology. 2008;28(3):403-412.

29. Flierl U, Bauersachs J, Schäfer A. Modulation of Platelet and Monocyte Function by the Chemokine Fractalkine (CX3CL1) in Cardiovascular Disease. Eur J Clin Invest. 2015;45(6):624-633.

30. von Hundelshausen P, Weber KS, Huo Y, et al. RANTES Deposition by Platelets Triggers Monocyte Arrest on Inflamed and Atherosclerotic Endothelium. Circulation. 2001;103(13):1772-1777.

31. Cuadrado MJ, Buendía P, Velasco F, et al. Vascular Endothelial Growth Factor Expression in Monocytes from Patients with Primary Antiphospholipid Syndrome. J Thromb Haemost. 2006;4(11):2461-2469.

32. Liberale L, Carbone F, Montecucco F, Sahebkar A. Statins reduce vascular inflammation in atherogenesis: A review of underlying molecular mechanisms. Int J Biochem Cell Biol. 2020 May;122:105735. doi:

10.1016/j.biocel.2020.105735. Epub 2020 Feb 29. PMID: 32126319.

33. Francisci D, Pirro M, Schiaroli E, et al. Maraviroc Intensification Modulates Atherosclerotic Progression in HIVSuppressed Patients at High Cardiovascular Risk. A Randomized, Crossover Pilot Study. Open Forum Infect Dis. 2019;6(4):ofz112.

34. Veenstra M, León-Rivera R, Li M, Gama L, Clements JE, Berman JW. Mechanisms of CNS Viral Seeding by HIV+ CD14+ CD16+ monocytes: Establishment and Reseeding of Viral Reservoirs Contributing to HIV-Associated Neurocognitive Disorders. mBio. Published online October 24, 2017.

35. Mondal S, Rangasamy SB, Roy A, Dasarathy S, Kordower JH, Pahan K. Low-dose Maraviroc, an Antiretroviral Drug, Attenuates the Infiltration of T-cells into the Central Nervous System and Protects the Nigrostriatum in Hemiparkinsonian Monkeys. The Journal of Immunology. 2019;202(12):3412-3422.

36. Liao Y, Chen S, Liu X, et al. Flow-Mediated Vasodilation and Endothelium Function in Children with Postural Orthostatic Tachycardia Syndrome. Am J Cardiol. 2010;106(3):378-382.

37. Gunning WT, Stepkowski SM, Kramer PM, Karabin BL, Grubb BP. Inflammatory Biomarkers in Postural Orthostatic Tachycardia Syndrome with Elevated G-Protein-Coupled Receptor Autoantibodies. J Clin Med. 2021;10(4):623. 
38. Poniatowski TA, Wojdasiewicz P, Krawczyk M, et al. Analysis of the Role of CX3CL2(Fractalkine) and its Receptor CX3CR1 in Traumatic Brain and Spinal Cord Injury: Insight into Recent Advances in Actions of Neurochemokine Agents. Mol Neurobiol. 2017;54(3):2167-2188.

39. Johansson PI, Sørensen AM, Perner A, et al. High sCD40L Levels Early After Trauma are Associated with Enhanced Shock, Sympathoadrenal Activation, Tissue and Endothelial Damage, Coagulopathy and Mortality. J Thromb Haemost. 2012;10(2):207-216.

40. Oude Nijhuis CSM, Vellenga E, Daenen SMGJ, Kamps WA, De Bont ESJM. Endothelial Cells Are Main Producers of Interleukin 8 through Toll-like Receptor 2 and 4 Signaling During Bacterial Infection in Leukopenic Cancer Patients. Clin Diagn Lab Immunol. 2003;10(4):558-563.

41. Sakoda K, Yamamoto M, Negishi Y, Liao JK, Node K, Izumi Y. Simvastatin Decreases IL-6 and IL-8 Production in Epithelial Cells. J Dent Res. 2006;85(6):520-523.

42. Cipriani S, Francisci D, Mencarelli A, et al. Efficacy of the CCR5 Antagonist Maraviroc in Reducing Early, RitonavirInduced Atherogenesis and Advanced Plaque Progression in Mice. Circulation. 2013;127(21):2114-2124.

43. Belge KU, Dayyani F, Horelt A, et al. The proinflammatory CD14+CD16+DR++ monocytes are a major Source of TNF. The Journal of Immunology. 2002;168(7):3536-3542.

44. Damås JK, Otterdal K, Yndestad A, et al. Soluble CD40 Ligand in Pulmonary Arterial Hypertension. Circulation. 2004;110(8):999-1005.

45. Rabinovici R, Sofronski MD, Borboroglu P, et al. Interleukin-2-Induced Lung Injury. The Role of Complement. Circ Res. 1994;74(2):329-335.

46. Kim SW, Kang HJ, Jhon M, et al. Statins and Inflammation: New Therapeutic Opportunities in Psychiatry. Front Psychiatry. 2019;0.

47. Giaquinto C, Mawela MP, Chokephaibulkit K, et al. Pharmacokinetics, Safety and Efficacy of Maraviroc in Treatment-Experienced Pediatric Patients Infected with CCR5-Tropic HIV-1. Pediatr Infect Dis J. 2018;37(5):459465.

48. Gulick RM, Fatkenheuer G, Burnside R, et al. Five-year Safety Evaluation of Maraviroc in HIV-1-infected Treatment-Experienced Patients. J Acquir Immune Defic Syndr. 2014;65(1):78-81.

49. Ayoub, Aymana; Alston, Sama; Goodrich, Jamesb; Heera, Jayvantb; Hoepelman, Andy IMc; Lalezari, Jacobd; Mchale, Marya; Nelson, Marke; van der Ryst, Elnaa; Mayer, Howardf Hepatic Safety and Tolerability in the Maraviroc Clinical Development Program, AIDS: November 13, 2010 - Volume 24 - Issue 17 - p 2743-2750

\section{Figures}




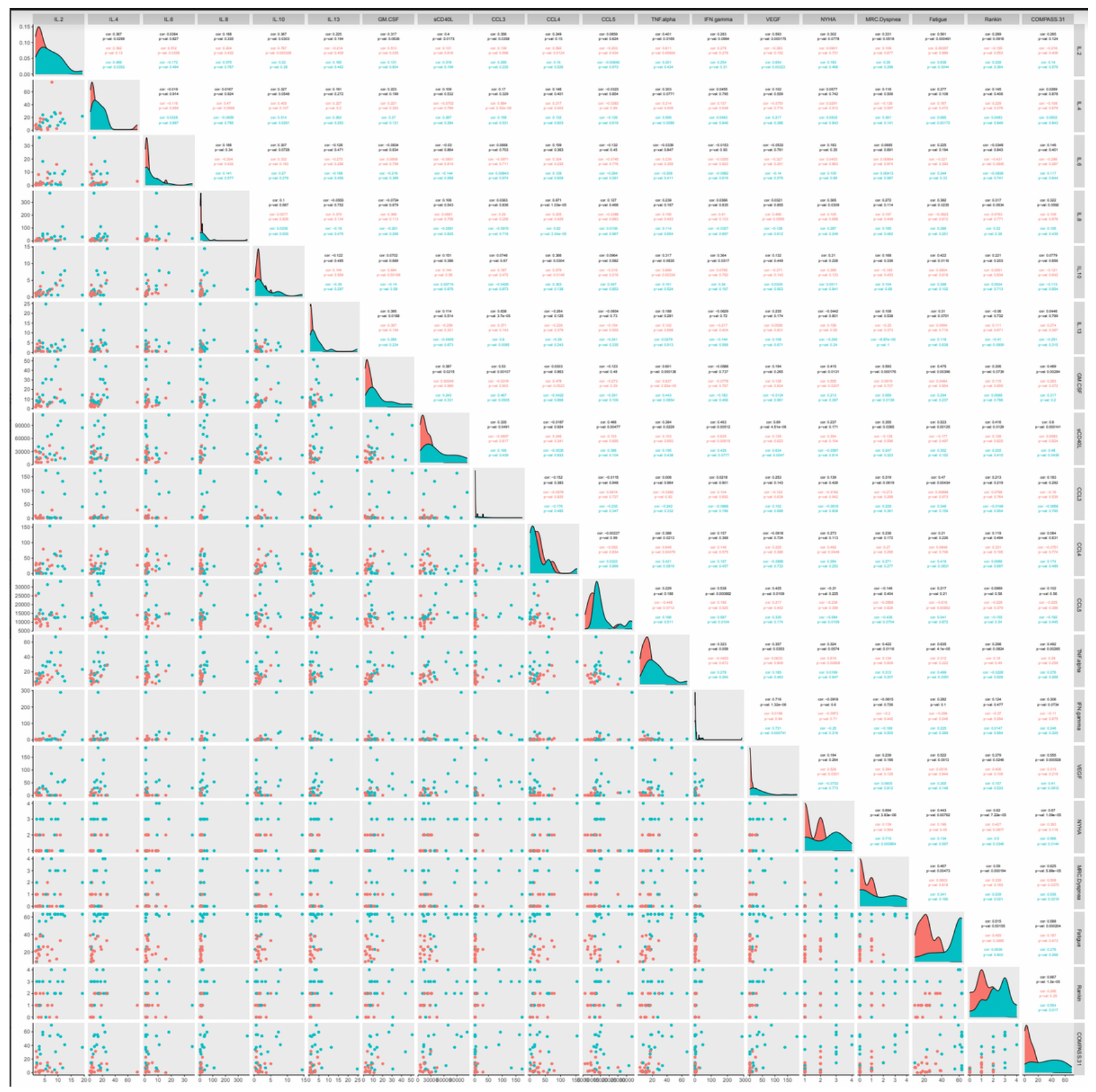

Figure 1

Correlation Matrix 


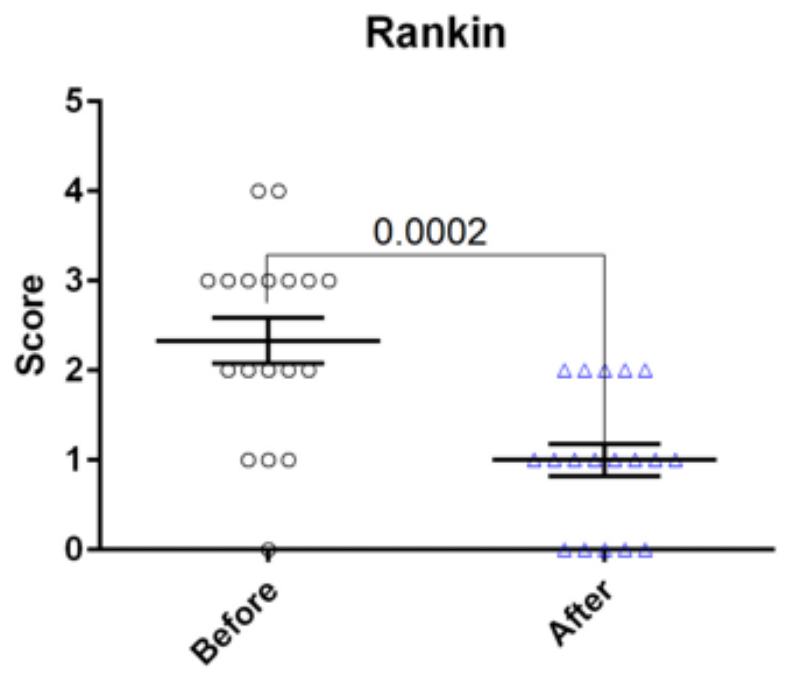

\title{
Biomarker Correlation
}

\author{
SCD40L CC-0.42, $\mathrm{P}=0.01$ \\ VEGF CC- $0.4, \quad P=0.02$
}

\section{Figure 2}

Modified Rankin Scale for Neurological Disability

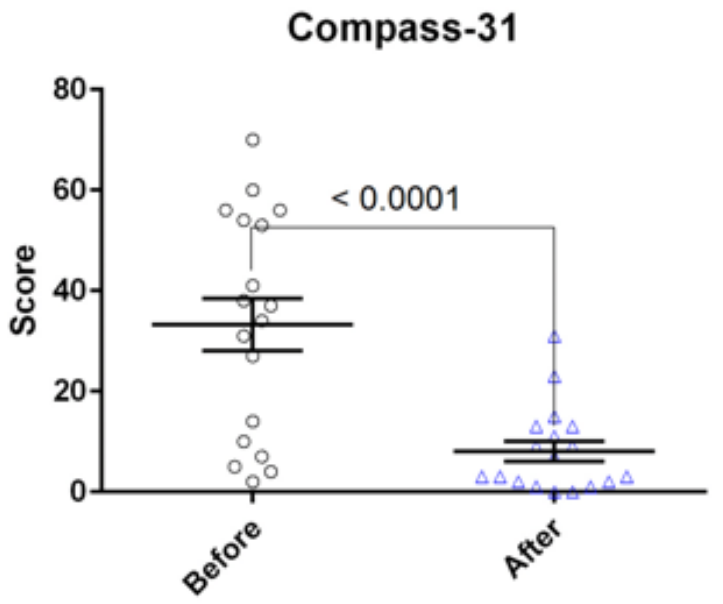

\section{Biomarker Correlation}

\author{
SCD40L CC-0.6, $\mathrm{P}=0.0001$ \\ VEGF CC-0.6, $P=0.0005$ \\ GM-CSF $\quad$ CC- $0.5 \quad P=0.002$
}

\section{Figure 3}

Composite Autonomic Symptom Scale 31 (COMPASS 31), 
NYHA

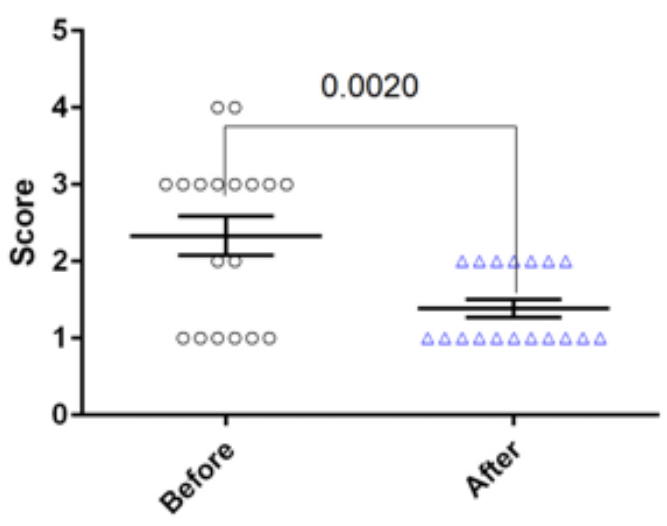

Figure 4

New York Heart Association Classification

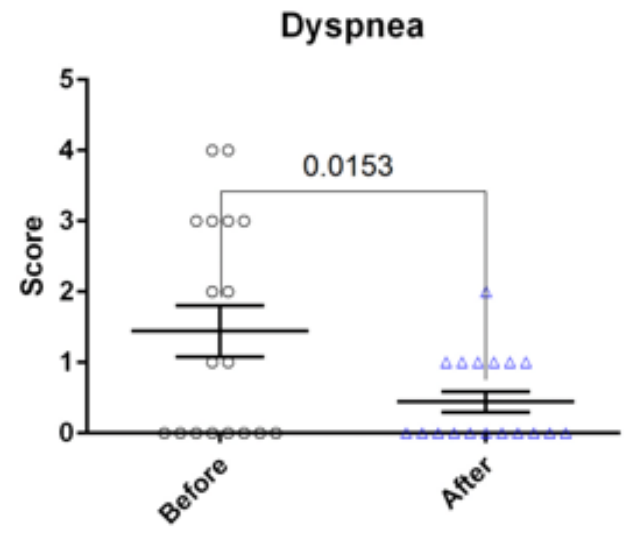

\section{Biomarker Correlation}

GM-CSF CC-0.4 P=0.01

IL-8

CC-0.4 P=0.03

\section{Biomarker Correlation}

\author{
SCD40L CC-0.4, P=0.04 \\ IL-2 CC-0.4, P=0.05 \\ GM-CSF CC-0.6 P=0.0002 \\ TNF $\quad$ CC-0.4 $\quad \mathrm{P}=0.01$
}

Figure 5

Medical Research Council (MRC) Dyspnea Scale 


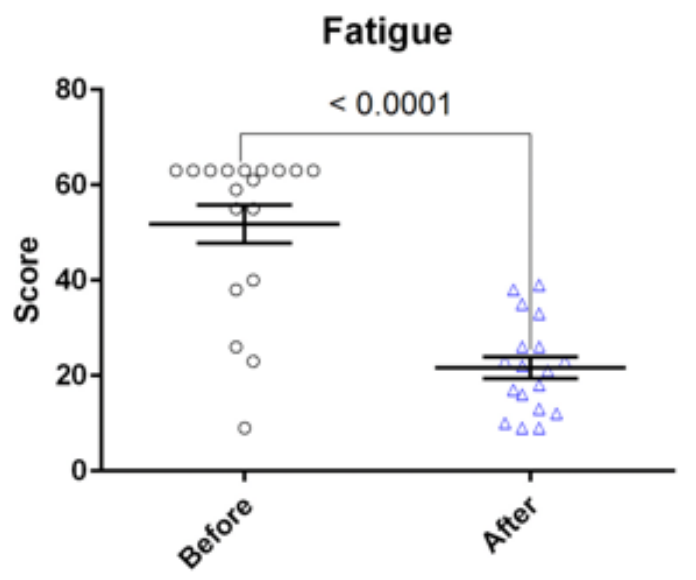

Figure 6

Fatigue Severity Score (FSS)

\section{Biomarker Correlation}

\author{
SCD40L CC-0.5, $P=0.001$ \\ IL-2 CC-0.6, $\quad \mathrm{P}=0.0005$ \\ GM-CSF CC-0.5 P=0.004 \\ TNF $\quad C C-0.7 \quad P=4 \times 10-5$ \\ VEGF $\quad$ CC- $0.5 \quad P=0.001$
}

